\title{
SHORT COMMUNICATION Pre-treatment microbial Prevotella-to-Bacteroides ratio, determines body fat loss success during a 6-month randomized controlled diet intervention
}

\author{
MF Hjorth ${ }^{1}$, HM Roager ${ }^{2}$, TM Larsen ${ }^{1}$, SK Poulsen ${ }^{1,3}$, TR Licht ${ }^{2}$, MI Bahl $^{2}$, Y Zohar $^{4}$ and A Astrup ${ }^{1}$
}

\begin{abstract}
On the basis of the abundance of specific bacterial genera, the human gut microbiota can be divided into two relatively stable groups that might have a role in personalized nutrition. We studied these simplified enterotypes as prognostic markers for successful body fat loss on two different diets. A total of 62 participants with increased waist circumference were randomly assigned to receive an ad libitum New Nordic Diet (NND) high in fiber/whole grain or an Average Danish Diet for 26 weeks. Participants were grouped into two discrete enterotypes by their relative abundance of Prevotella spp. divided by Bacteroides spp. ( $P / B$ ratio) obtained by quantitative $P C R$ analysis. Modifications of dietary effects of pre-treatment $P / B$ group were examined by linear mixed models. Among individuals with high $P / B$ the NND resulted in a $3.15 \mathrm{~kg}(95 \%$ confidence interval $(\mathrm{Cl}): 1.55 ; 4.76$, $P<0.001)$ larger body fat loss compared with $A D D$, whereas no differences was observed among individuals with low $P / B(0.88 \mathrm{~kg}$ $(95 \% \mathrm{Cl}:-0.61 ; 2.37, P=0.25))$. Consequently, a $2.27 \mathrm{~kg}(95 \% \mathrm{Cl}: 0.09 ; 4.45, P=0.041)$ difference in responsiveness to the diets were found between the two groups. In summary, subjects with high $P / B$ ratio appeared more susceptible to lose body fat on diets high in fiber and whole grain than subjects with a low $P / B$ ratio.
\end{abstract}

International Journal of Obesity (2018) 42, 580-583; doi:10.1038/ijo.2017.220

\section{INTRODUCTION}

The composition of the gut microbiota in rodents has been shown to affect the efficacy of energy harvest from feed ${ }^{1}$ and to influence the secretion of gastrointestinal hormones affecting appetite. ${ }^{2}$ Therefore, it seems as if the human gut microbiota has the potential to have a pivotal role in personalized nutrition. ${ }^{3,4}$

Clustering of the human gut microbiota, designated enterotypes, was first described in 2011.5 The Bacteroides-driven enterotype is reported to be predominant in individuals consuming more protein and animal fat (western diet), whereas the Prevotella-driven enterotype appears predominant in subjects consuming more carbohydrate and fiber. ${ }^{6-8}$ That said, the enterotype of an individual has been shown to remain rather stable. ${ }^{6,7,9}$ A limited number of studies have related microbial enterotypes to health markers; ${ }^{8-10}$ however, body fat change during a randomized clinical trial is not one of them.

Therefore, as a proxy for enterotypes, we studied pre-treatment Prevotella-to-Bacteroides $(P / B)$ ratio as a prognostic marker for successful body fat loss on two diets differing greatly in dietary fiber and whole-grain content.

\section{MATERIALS AND METHODS}

In total 181 participants with increased waist circumference were randomly assigned to receive an ad libitum New Nordic Diet (NND) or a control diet for 26 weeks of which a subgroup of 62 subjects were randomized to collect fecal samples. The macronutrient composition of the NND was based on Nordic Nutrition
Recommendations, whereas the control diet was designed to match the macronutrient composition of an Average Danish Diet (ADD). ${ }^{11}$ The NND is a whole-food approach characterized by being very high in dietary fiber, whole grain, fruit and vegetables. ${ }^{12}$ For both groups, food and beverages were provided from a study shop free of charge throughout the intervention period. ${ }^{12}$ Pre-intervention fasting blood samples were drawn from where fasting glucose and insulin were analyzed. Height was measured at baseline and body weight was measured at randomization and week 2, 4, 8, 12, 16, 20, 24 and 26. Furthermore, waist circumference and fat mass (using DEXA) were measured at randomization, week 12 and 26 . Fecal samples were collected at baseline and the relative abundance of Prevotella spp. and Bacteroides spp. was determined using genera-specific quantitative PCR targeting the bacterial $16 \mathrm{~S}$ ribosomal gene regions as previously described. ${ }^{9}$ As previously reported by Roager et al., ${ }^{9}$ this resulted in a clear bimodal separation of subjects based on the log Prevotella spp. to Bacteroides spp. ratio, in the following designated low $P / B$ $(<0.01)$ or high $P / B(>0.01)$. In eight samples, Prevotella spp. was below the detection limit and were classified as low $P / B$ in the main analysis and excluded in a sensitivity analysis. Regardless of randomization status, after the completion of the first 26 weeks, all participants were instructed to follow the NND for an additional year (weight measured after 52 and 78 weeks) without any provision of food ${ }^{13}$ to investigate the diets in a real life setting. The study was approved by the ethical committee of the Capital Region of Denmark (reference $\mathrm{H}-3-2010-058$ ) and registered at clinicaltrials.gov as NCT01195610.

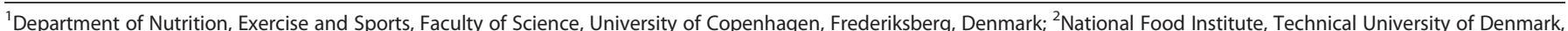

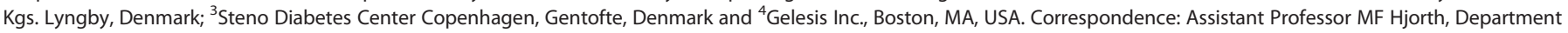
of Nutrition, Exercise and Sports, Faculty of Science, University of Copenhagen, Rolighedsvej 26, Frederiksberg 1958 Denmark. 
Statistics

Baseline characteristics were summarized as mean \pm s.d., median (interquartile range) or proportions (\%) and differences between $P / B$ groups as well as dietary groups were tested using a parametric (some variables transformed before analysis) or nonparametric two-sample test or Pearson's $x^{2}$ test.

The differences in body fat (as well as weight and waist circumference) change from baseline between P/B groups on the two diets were analyzed by means of linear mixed models using all available measurements. The linear mixed models included the three-way interaction between $\operatorname{diet} \times$ time $\times P / B$ group strata as well as all nested two-way interactions and main effects and comprised additional fixed effects including age, gender, baseline $\mathrm{BMI}$, baseline fasting glucose and insulin as well as random effects for subjects. Results are shown as mean change from baseline with $95 \%$ confidence interval $(\mathrm{Cl})$. The level of significance was set at $P<0.05$ and statistical analyses were conducted using STATA/ SE 14.1 (Houston, TX, USA).

\section{RESULTS}

The NND compared to ADD was higher in dietary fiber (43.3 vs $28.6 \mathrm{~g} / 10 \mathrm{MJ})$, higher in protein (18.1 vs $16.4 \%$ ), lower in fat (30.4 vs $33.8 \%$ ) (all $P<0.001$ ) without differing in available carbohydrates (46.4 vs $45.3 \%$; $P=0.081$ ).

No differences in baseline characteristics were found between individuals characterized as high and low $P / B$ (all $P \geqslant 0.09$ ) (Table 1). Among individuals with a high $P / B$ ratio, the NND diet resulted in a $3.15 \mathrm{~kg}(95 \% \mathrm{Cl}: 1.55 ; 4.76, P<0.001)$ larger body fat loss compared to ADD after 26 weeks, whereas no difference in body fat loss was observed between NND and ADD among individuals with low $P / B(0.88 \mathrm{~kg}(95 \% \mathrm{Cl}:-0.61 ; 2.37, P=0.25))$. Consequently, a $2.27 \mathrm{~kg}(95 \% \mathrm{Cl}: 0.09 ; 4.45, P=0.041)$ difference in responsiveness to the diets was found between the $P / B$ groups, which came from difference in response to NND $(P=0.04)$ and not ADD $(P=0.41)$ between the $P / B$ groups (Table 2). Similar differences in responsiveness to the diets were found for waist circumference $(3.95 \mathrm{~cm}(95 \% \mathrm{Cl}: 0.34 ; 7.55, P=0.032))$ and were borderline significant for body weight $(2.33 \mathrm{~kg}(95 \% \mathrm{Cl}$ : -0.15 ; 4.80, $P=0.065$ )) (Table 2 ). The sensitivity analysis revealed larger differences (Table 2).

During the 1 year follow-up period, subjects with the high $P / B$ ratio changing from $A D D$ to being recommended NND maintained their weight $(-1.23(95 \% \mathrm{Cl}:-2.81 ; 0.36, n=9, P=0.13))$, whereas subjects with the low $P / B$ ratio changing from $A D D$ to being recommended NND regained $2.76 \mathrm{~kg}(95 \% \mathrm{Cl}: 1.27 ; 4.24$, $n=11, P<0.001)$. Consequently, a $3.99 \mathrm{~kg}(95 \% \mathrm{Cl}: 1.82 ; 6.15$, $P<0.001)$ difference in responsiveness to the NND were found between $P / B$ groups during the 1 year follow-up. This difference was $5.41 \mathrm{~kg}(95 \% \mathrm{Cl}: 3.12 ; 7.69, P<0.001)$ in the sensitivity analysis.

\section{DISCUSSION}

We identified pre-treatment $P / B$ ratio as an important biomarker associated with body fat loss in subjects consuming an ad libitum diet rich in fiber and whole grain. Thus, overweight and obese participants with high $P / B$ ratio appeared more responsive to fiber and whole grain than individuals with low $P / B$ ratio. This was further supported by similar findings for waist circumference and body weight.

Using the entire sample of 181 subjects, we have previously reported the overall weight-loss difference between the NND and ADD to be $3.2 \mathrm{~kg}^{12}$ Interestingly, this difference between diets could mainly be attributed to subjects with the high $P / B$ ratio, and the health-promoting aspects of the NND in terms of body-weight regulation, therefore, mainly seems to apply in a subset of the population.

Previously, baseline total cholesterol has been found to be borderline higher $(P=0.08)^{9}$ and LDL cholesterol to be lower ${ }^{8}$ among the Prevotella-driven enterotype. Furthermore, the enterotypes have been found to impact in vitro fermentation profiles of short chain fatty acids from the same carbohydrate substrates differentially, with the Prevotella-driven enterotype having higher total short chain fatty acid production. ${ }^{3}$ In vitro, some of these short chain fatty acids have been shown to stimulate the secretions of gastrointestinal hormones affecting appetite. ${ }^{2}$ Finally, in an observational study of 1632 women, the abundance of Bacteroides spp. was associated with weight gain, whereas dietary fiber intake was found partly to modify the association between microbiome diversity and weight gain. ${ }^{14}$

The distinction of enterotypes as discrete clusters has recently been challenged by studies suggesting that enterotype distribution is continuous and that further information may be masked within these enterotype clusters. ${ }^{15,16}$ From our analysis, we cannot determine specific bacterial species responsible for the dietary effects that we observe but only highlight the relative abundance of Prevotella spp. (genus) as important in the classification of microbiota profiles. Nevertheless, our sensitivity analysis indicates that subjects with Prevotella spp. below the detection limit behave different than subjects in the low $P / B$ ratio group.

The increased responsiveness of the high $P / B$ group to the NND, rich in fruits, vegetables, dietary fibers and whole grains, is supported by previous studies showing an association between the Prevotella-driven enterotype and a carbohydrate-based diet more typical of agrarian societies. ${ }^{6}$ However, only two individuals switched $P / B$ ratio group during this 6-month dietary intervention with NND or $\mathrm{ADD}^{9}{ }^{9}$ which is consistent with the literature

Table 1. Baseline characteristics of the study populations stratified by enterotype $(n=62)$

\begin{tabular}{|c|c|c|c|}
\hline Age (year) & $41.9(30.4 ; 56.7)$ & $47.5(33 ; 55.6)$ & 0.33 \\
\hline Gender (\%female/male) & $64.3 / 35.7$ & $69.2 / 30.8$ & 0.70 \\
\hline Body mass index $\left(\mathrm{kg} \mathrm{m}^{-2}\right)$ & $31.0 \pm 4.7$ & $29.0 \pm 4.4$ & 0.09 \\
\hline Body fat (\%) & $40.5 \pm 6.4$ & $38.9 \pm 7.1$ & 0.36 \\
\hline Fasting glucose $\left(\mathrm{mmol} \mathrm{I}^{-1}\right)$ & $5.34 \pm 0.51$ & $5.19 \pm 0.40$ & 0.20 \\
\hline Fasting insulin $\left(\mathrm{pmol}^{-1}\right)$ & $54.5(41 ; 78)$ & $47.5(35 ; 74)$ & 0.14 \\
\hline Prevotella-to-Bacteroides ratio & $0.28(0.11 ; 7.50)$ & $0.00007(0.00001 ; 0.00026)$ & \\
\hline
\end{tabular}




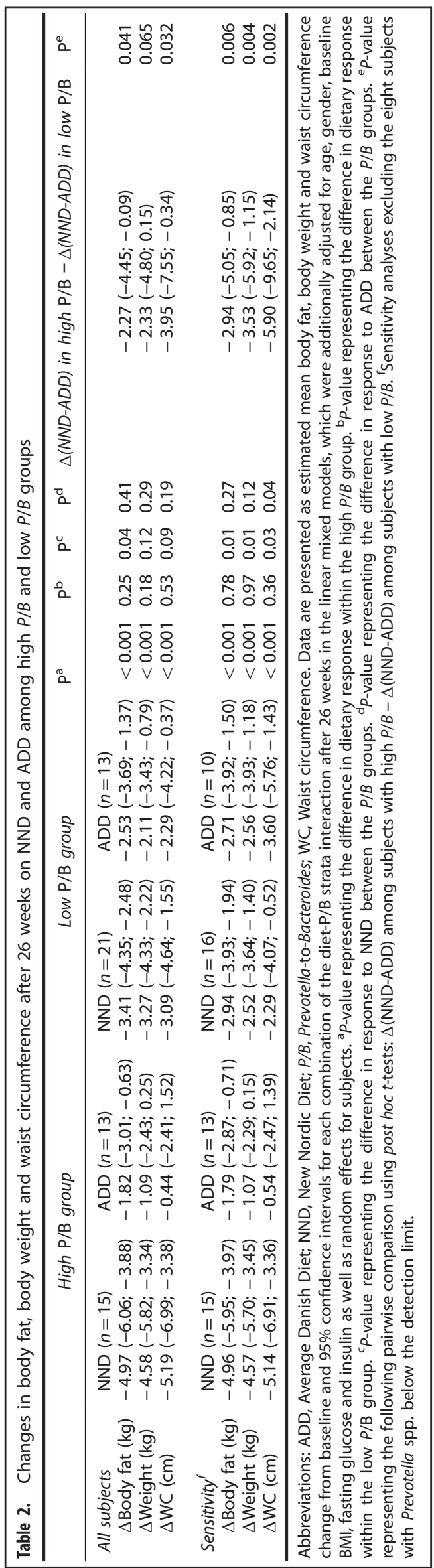

indicating that intestinal microbial communities are resilient and difficult to change through dietary interventions $s^{6,7,9}$ unless extreme changes, such as complete removal of carbohydrates from the diet, are introduced. ${ }^{17}$

Mechanisms involved could be efficacy of energy harvest from different foods, ${ }^{1}$ differences in fiber-utilization capacity, ${ }^{3}$ gut-brain signaling of behavior ${ }^{18}$ and the secretion of gastrointestinal hormones affecting appetite. ${ }^{2,10}$ Recently, dietary fiber-induced improvements in post-prandial blood glucose and insulin were found to be positively associated with the abundance of Prevotella. ${ }^{19}$ Therefore, the recent breakthrough in personalized nutrition, showing the importance of pre-treatment fasting glucose and insulin to determine the optimal diet for weight management, ${ }^{20}$ might also be linked to gut microbiota profiles. We therefore adjusted for a number of potential confounders including fasting glucose and insulin. However, independent of the mechanisms, the $P / B$ ratio may serve as a biomarker to predict future weight-loss success on specific diets.

In summary, we identified pre-treatment $P / B$ ratio as an important biomarker associated with dietary body fat change on ad libitum high fiber diets. Thus, individuals with a high $P / B$ ratio were more susceptible to body fat loss on a diet rich in fiber and whole grain compared to an average Danish diet, whereas no difference in body fat loss was observed in individuals with a low $P / B$ ratio.

\section{CONFLICT OF INTEREST}

$\mathrm{MFH}, \mathrm{YZ}$ and $\mathrm{AA}$ are co-inventers on a pending provisional patent application on the use of biomarkers for prediction of weight-loss responses. The work reported in this manuscript was funded by grants from Gelesis Inc. The remaining authors declare no conflict of interest.

\section{ACKNOWLEDGEMENTS}

The overall study was supported by the Nordea Foundation (Grant No. 02-2010-0389) and sponsors who provided foods to the shop. A full list of food sponsors is available at the study website (www.foodoflife.dk/shopus). The present stratified analysis was supported by a grant from Gelesis Inc.

\section{AUTHOR CONTRIBUTIONS}

MFH and AA designed research; MFH performed statistical analysis and wrote the first draft. HMR, TRL and MIB conceived, designed and performed the part of the $P / B$ ratio analyses reported in a previous publication. ${ }^{9}$ All authors have contributed to the discussion of analyses, reviewed the manuscript critically and approved the final manuscript.

\section{REFERENCES}

1 Turnbaugh PJ, Ley RE, Mahowald MA, Magrini V, Mardis ER, Gordon JI. An obesityassociated gut microbiome with increased capacity for energy harvest. Nature 2006; 444: 1027-1131.

2 Tolhurst G, Heffron H, Lam YS, Parker HE, Habib AM, Diakogiannaki E et al. Short-chain fatty acids stimulate glucagon-like peptide-1 secretion via the G-protein-coupled receptor FFAR2. Diabetes 2012; 61: 364-371.

3 Chen T, Long W, Zhang C, Liu S, Zhao L, Hamaker BR. Fiber-utilizing capacity varies in Prevotella- versus Bacteroides-dominated gut microbiota. Sci Rep 2017; 7: 2594.

4 Zeevi D, Korem T, Zmora N, Israeli D, Rothschild D, Weinberger A et al. Personalized nutrition by prediction of glycemic responses. Cell 2015; 163: 1079-1094.

5 Arumugam M, Raes J, Pelletier E, Le Paslier D, Yamada T, Mende DR et al. Enterotypes of the human gut microbiome. Nature 2011; 473: 174-180.

6 Wu GD, Chen J, Hoffmann C, Bittinger K, Chen YY, Keilbaugh SA et al. Linking long-term dietary patterns with gut microbial enterotypes. Science 2011; 334: 105-108.

7 Lim MY, Rho M, Song Y, Lee K, Sung J, Ko G. Stability of gut enterotypes in Korean monozygotic twins and their association with biomarkers and diet. Sci Rep 2014; 4: 7348. 
8 de Moraes AC, Fernandes GR, da Silva IT, Almeida-Pititto B, Gomes EP, da Costa Pereira $A$ et al. Enterotype may drive the dietary-associated cardiometabolic risk factors. Front Cell Infect Microbiol 2017; 7: 47.

9 Roager HM, Licht TR, Poulsen SK, Larsen TM, Bahl MI. Microbial enterotypes, inferred by the prevotella-to-bacteroides ratio, remained stable during a 6-month randomized controlled diet intervention with the New Nordic Diet. Appl Environ Microbiol 2014; 80: 1142-1149.

10 Kang C, Zhang Y, Zhu X, Liu K, Wang X, Chen M et al. Healthy subjects differentially respond to dietary capsaicin correlating with specific gut enterotypes. J Clin Endocrinol Metab 2016; 101: 4681-4689.

11 Pedersen AN, Fagt S, Groth MV, Christensen T, Biltoft-Jensen AP, Matthiessen J et al. Danskernes Kostvaner 2003-2008: Hovedresultater. DTU Fødevareinstituttet: Søborg, Denmark, 2010.

12 Poulsen SK, Due A, Jordy AB, Kiens B, Stark KD, Stender S et al. Health effect of the New Nordic Diet in adults with increased waist circumference: a 6-mo randomized controlled trial. Am J Clin Nutr 2014; 99: 35-45.

13 Poulsen SK, Crone C, Astrup A, Larsen TM. Long-term adherence to the New Nordic Diet and the effects on body weight, anthropometry and blood pressure: a 12-month follow-up study. Eur J Nutr 2015; 54: 67-76.

14 Menni C, Jackson M, Pallister T, Steves C, Spector T, Valdes A. Gut microbiome diversity and high-fibre intake are related to lower long-term weight gain. Int J Obes 2017; 41: 1099-1105.

15 Knights D, Ward TL, McKinlay CE, Miller H, Gonzalez A, McDonald D et al. Rethinking 'enterotypes'. Cell Host Microbe 2014; 16: 433-437.
16 Gorvitovskaia A, Holmes SP, Huse SM. Interpreting Prevotella and Bacteroides as biomarkers of diet and lifestyle. Microbiome 2016; 4: 15.

17 Andrés $M$, Ana D, Juan José A, Amparo L. Effect of dietary carbohydrate restriction on an obesity-related Prevotella-dominated human faecal microbiota. Metagenomics 2013; 2: 235722.

18 Mayer EA, Tillisch K, Gupta A. Gut/brain axis and the microbiota. J Clin Invest 2015; 125: 926-38.

19 Kovatcheva-Datchary P, Nilsson A, Akrami R, Lee YS, De Vadder F, Arora T et al. Dietary fiber-induced improvement in glucose metabolism is associated with increased abundance of Prevotella. Cell Metab 2015; 22: 971-82.

20 Hjorth MF, Ritz C, Blaak EE, Saris WHM, Langin D, Poulsen SK et al. Pre-treatment fasting plasma glucose and insulin modify dietary weight loss success: results from three randomized clinical trials. Am Clin Nutr 2017; 106: 499-505.

This work is licensed under a Creative Commons Attribution 4.0 International License. The images or other third party material in this article are included in the article's Creative Commons license, unless indicated otherwise in the credit line; if the material is not included under the Creative Commons license, users will need to obtain permission from the license holder to reproduce the material. To view a copy of this license, visit http://creativecommons.org/licenses/ by/4.0/

(c) The Author(s) 2018 